\title{
Effective Factors on Sharp Score in Patients with Rheumatoid Arthritis: A Retrospective Study
}

\section{Jianting Wen}

Anhui University of Traditional Chinese Medicine - Meishan Road Campus: Anhui University of

Traditional Chinese Medicine

\section{Jian Liu ( $\square$ liujianahzy@126.com )}

First Affiliated Hospital of Anhui University of Chinese medicine https://orcid.org/0000-0003-3101-

7553

\section{Xin Wang}

Anhui University of Traditional Chinese Medicine - Meishan Road Campus: Anhui University of

Traditional Chinese Medicine

Jie Wang

Anhui University of Traditional Chinese Medicine - Meishan Road Campus: Anhui University of

Traditional Chinese Medicine

\section{Research article}

Keywords: Sharp Score, Rheumatoid arthritis, Effective factors, Retrospective study

Posted Date: February 16th, 2021

DOI: https://doi.org/10.21203/rs.3.rs-241593/v1

License: (c) (i) This work is licensed under a Creative Commons Attribution 4.0 International License.

Read Full License 


\title{
Effective factors on sharp score in patients with rheumatoid arthritis:
}

\section{a retrospective study}

\author{
Jianting Wen ${ }^{1,3}$, Jian Liu $^{2,3^{*}}$, Xin Wang ${ }^{1}$, Jie Wang ${ }^{1}$ \\ ${ }^{1}$ Anhui University of Traditional Chinese Medicine, Hefei 230031, Anhui Province, \\ China \\ ${ }^{2}$ Department of Rheumatology and Immunology, First Affiliated Hospital of Anhui \\ University of Traditional Chinese Medicine, Hefei 230038, Anhui Province, China \\ ${ }^{3}$ Institute of Rheumatology, Anhui Academy of Traditional Chinese Medicine, Hefei \\ 230012, Anhui Province, China \\ * Correspondence to: Jian Liu, Ph.D, Department of Rheumatology and Immunology, \\ First Affiliated Hospital of Anhui University of Traditional Chinese Medicine, Hefei \\ 230038, Anhui Province, China. E-mail address: liujianahzy@126.com. Tel: +86 0551 \\ 62838582, Fax: +86 055162821605 .
}

\begin{abstract}
Background: This study aims to describe the association between Sharp Score and clincial indexes, bone metabolism indexes, Disease Activity Score (DAS28) and sociodemographic factors in rheumatoid arthritis (RA).

Methods: Data were were collected from the HIS (hospital information system), a national inpatient database in China, with information on the patients hospitalized during the period from 2012 to 2019. The association between Sharp Score and effective factors were identified using multinomial logistic regression and association rule mining $(\mathrm{ARM})$.
\end{abstract}

Results: Three thousand eight hundred and forty patients were included: $82.66 \%$ males, 17.34\% females, mean (SD) age 56.95 (12.68) years and symptom duration 3.45 (1.09) years. Spearman correlation analysis and Association rules analysis showed that there were significant positive correlations between Sharp Score and effective factors. Logistic regression analysis presented that erythrocyte sedimentation rate (ESR), high-sensitivity C-reactive protein (CRP), rheumatoid factor (RF) were 
risk factors of Sharp Score. In the analysis of individual outcomes, sex, age, symptom duration, DSA28 score, RF, ever drinker, radiographic grading of hands were influencents of Sharp Score.

Conclusion: Sharp Score should be taken into consideration in formulating treatment strategies in RA.

Keywords: Sharp Score, Rheumatoid arthritis, Effective factors, Retrospective study

\section{Background}

Rheumatoid arthritis (RA) is a chronic inflammatory disease characterized by synovial membrane inflammation ${ }^{1,2}$. Erosive joint damage and bone destruction are the most common manifestation of RA, which might induce ankylosis, malformation, even loss of normal joint function ${ }^{3,4}$. Current goals of treatment in RA include achieving disease remission, reducing functional disability as well as minimizing pain $^{5,6}$. Erosions are the hallmark of bone destruction in $\mathrm{RA}^{7,8}$. Controlling joint destruction and bone destruction have become the major objective for treating RA, because radiographic joint damage correlates strongly with long-term functional decline in RA patients ${ }^{9,10}$. Radiographic grading of hands is the most commonly used method for the evaluation of different levels of bone erosion in clinical practice ${ }^{11,12}$. Bone erosion score (vdH Sharp Score) has also been used to measure morphological parameters that quantify the bone erosion and bone destruction, giving useful information for early detection and early treatment of $\mathrm{RA}^{13,14}$. Few studies to date have studied the overall impact of RA on Sharp Score and its effective factors. In addition, there has been no study that looks for effective factors associated with Sharp Score of RA on the basis of large data by doing the mining and analysis of this data.

This study retrospectively analyzed the clinical data of enrolled patients to investigate the value of Sharp Score and its effective factors in RA. The Spearman correlation analysis, Association rules analysis and Logistic regression analysis are methods of analysis that allows for the identification of risk factors associated with Sharp Score. Using these three methods, this study aims to: (a) Sharp Score exhibits diagnostic value for RA; and (b) analyze the role of effective factors as determinants 
of Sharp Score in RA.

\section{Methods}

\section{Patients and study design}

A total of 3840 RA patients were included in this retrospective study, which were obtained from the First Affiliated hospital of Anhui University of Traditional Chinese Medicine since January 2012. All the subjects fulfilled the 2010 ACR/EULAR (American College of Rheumatology/European League Against Rheumatism) criteria for the classification of $\mathrm{RA}^{15}$. Sharp Score, demographic data, lifestyle factors, clinical parameters and laboratory data were determined by clinical examination or review of electronic medical records. The study was conducted in accordance with the Declaration of Helsinki. Informed consent was obtained from all patients and ethics approval was obtained from the Ethics Committee of the First Affiliated hospital of Anhui University of Traditional Chinese Medicine.

\section{Data collection and measures}

All RA patients data were recorded soon after hospitalization, which including the details regarding patients' past medical, personal, and family histories. Besides this, RA-related data (age, sex, marital status, health insurance status, education level, employment status, and housing status, hypertension, hyperlipidaemia, smoking history, alcohol consumption history, treatment-condition) was collected from medical records. On the second day of hospitalization, patients general condition was monitored via vital signs, X-ray radiography of both hands, Sharp Score, blood biochemistry tests, bone metabolism indicators, DAS28 score.

X-ray radiography of both hands were performed to assess the extent of joint destruction, bone erosion and joint space narrowing. The Sharp Score of each patients was calculated by 2 independent blinded radiologists. Each joint bone erosion was scored as follows: $0=$ normal; $1=$ cell infiltration with no signs of joint erosion; $2=$ inflammation with the presence or erosions limited to discrete foci; and $3=$ severe and extensive joint erosion with loss of architecture. Each joint stenosis was scored as follows: $0=$ normal; 1 =focal or doubtful narrowing; $2=$ generalized narrowing $<50 \%$; 
$3=$ generalized narrowing $>50 \%$ or subluxation; $4=$ complete luxation or bony ankylosis.

Clinical indexes: ESR, CRP, RF, anti-cyclic citrullinated peptide antibody (CCP), immunoglobulins A (IGA), immunoglobulin G (IGG), immunoglobulin M (IGM), complement 3 (C3), and complement 4 (C4), DAS28, bone alkaline phosphatase (BALP), osteocalcin (OC), Osteoprotegerin (OPG), receptor activator for nuclear factor- $\mathrm{K}$ B ligand (RANKL).

\section{Statistical analysis}

Normally distributed variables were presented as the mean values (standard deviation), nonnormally distributed variables were presented as median values $(p 50, p 75)$, and count data were presented as the number of cases (\%). We conducted univariate analyses on important covariates, including age, gender, CCP or RF positivity, ESR, CRP, IGA, IGG, IGM, C3 and C4. Continuous variables with a normal distribution were compared using Student's t-test, while non-normally distributed variables were compared using the Wilcoxon rank sum test. Categorical variables were evaluated using Fisher's exact test. The correlations between Sharp Score and clinical indexes were analyzed by Spearman's correlation coefficients. In addition, we constructed a binary linear regression model to determine the association of important covariates with Sharp Score. Statistical tests were performed in Excel or in GraphPad Prism version 8.0.

The indicators rise was set to "T", while the indicators decline was set to "F". The Aprior module of SPSS Clementine 11.1 software was used to analyze the correlation between observation indicators. The most famous association rule is the Apriori algorithm, which aims to find out the relationship between items in a data set, also known as shopping blue analysis ${ }^{16}$. In our data, each drug was treated as a variable. The formulae were as follows: 


$$
\begin{array}{r}
\operatorname{support}(X \rightarrow Y)=\sigma \frac{(X \cup Y)}{N}, \\
\text { counfidence }(X \rightarrow Y)=\sigma \frac{(X \cup Y)}{\sigma(X)}, \\
\operatorname{lift}(X \rightarrow Y)=\text { counfidence } \frac{(X \rightarrow Y)}{\sigma(Y)},
\end{array}
$$

where $X \rightarrow Y$ is an association rule, $X$ (left-hand side [LHS]) and $Y$ (right-hand side [RHS]) represent the set of herb items, $\sigma(X)$ is the frequency of itemset $X, X \cup Y$ is the union of itemset $X$ and $Y, \sigma(X \cup Y)$ is the frequency with which itemset $X$ and itemset $Y$ appear together, $\operatorname{support}(X \rightarrow Y)$ is the frequency with which $X$ and $Y$ appear together, and confifidence $(X \rightarrow Y)$ is the probability that itemset $Y$ appears in the presence of $X$. The lift is the ratio of the probability of itemset $Y$ appearing in the presence of $X$ to the frequency of $Y$. Support and confifidence are often used to eliminate meaningless combinations; lift is the validity of the rules.

\section{Results}

\section{Characteristics of the study population}

The study sample was composed of 3840 RA patients, with a mean age of 56.95 years (standard deviation 12.68, range $18-95$ ) and of whom $82.66 \%$ were female. The median number of Sharp Score was 20.00 (IQR: 7.00, 56.00). 3003 (78.15\%) were seropositive for either RF and 3636 (94.68\%) were seropositive for CCP. The mean (SD) symptom duration was 3.45 (1.09) years and the mean (SD) disease duration was 6.92 (1.20) years. The main characteristics of the study population are detailed in Table 1.

Table 1. Characteristics of study population $(n=3840)$.

\begin{tabular}{lll}
\hline Quantitative Variables & Mean & Standard Deviation \\
\hline Age (years) & 56.95 & 12.68 \\
Symptom duration (years) & 3.45 & 1.09 \\
Disease duration (years) & 6.92 & 1.20 \\
BMI $(\mathrm{Kg} / \mathrm{m} 2)$ & 22.80 & 4.51 \\
\hline
\end{tabular}


Tender joint count, 0-28

Sharp Score

$\leqslant 0$ score

$>0$ score, $\leqslant 50$ score

$>50$ score

Gender

Male

Female

Ever smoker

Ever drinker

RF positivity

CCP positivity

Presence of radiographic erosions

Prenisolone use

DMARD treatment (at baseline)

DMARD-naive

MTX monotherapy

Non-MTX csDMARD

Combination csDMARD

Education status

None or primary

Secondary or vocational

Tertiary

Housing status

Private housing

Government housing

Employment status

Currently employed

Unemployment, retired or homemaker

Marital status

Currently married

Single, divorced or widowed

Sharp Score

DAS28 score

ESR $(\mathrm{mm} / \mathrm{h})$

CRP (mg/L)

$\mathrm{RF}(\mathrm{U} / \mathrm{ml})$

$\mathrm{CCP}(\mathrm{U} / \mathrm{ml})$

IGA $(g / L)$

IGG $(\mathrm{g} / \mathrm{L})$

IGM $(\mathrm{g} / \mathrm{L})$
10.73

Subjects

5.63

Percentage

$\begin{array}{ll}111 & 2.89 \\ 2674 & 69.64 \\ 1055 & 27.47\end{array}$

666

17.34

3174

82.66

458

11.93

582

15.16

3001

78.15

3636

94.68

2162

56.32

2150

55.98

3017

78.56

1368

35.62

888

23.13

905

23.56

1545

40.23

1740

45.31

555

14.45

2397

62.42

1443

37.58

1735

45.18

2105

54.82

3657

95.23

183

4.77

Interquartile range (IQR)

$20.00(7.00,56.00)$

$5.50(4.00,7.28)$

$42.00(22.00,68.00)$

$15.82(3.61,41.49)$

79.15 (19.90, 195.05)

$132.98(25.00,402.67)$

$2.47(1.87,3.27)$

$13.45(11.00,16.40)$

$1.35(0.91,1.68)$ 


\begin{tabular}{ll}
\hline C3 $(\mathrm{g} / \mathrm{L})$ & $112.45(96.50,129.30)$ \\
C4 $(\mathrm{g} / \mathrm{L})$ & $24.7(19.20,30.80)$ \\
BALP $(\mathrm{ng} / \mathrm{ml})$ & $660.13(431.37,851.60)$ \\
BGP $(\mathrm{ng} / \mathrm{ml})$ & $3479.49(2871.84,4825.99)$ \\
OPG $(\mathrm{ng} / \mathrm{ml})$ & $117.20(948.12,1338.62)$ \\
RANKL $(\mathrm{ng} / \mathrm{ml})$ & $1085.40(955.51,1322.46)$ \\
\hline
\end{tabular}

\section{Spearman correlation analysis of Sharp Score and clincial indexes}

To determine whether correlations existed between Sharp Score and clincial indexes, a Spearman correlation test was performed. Age, ESR, CRP, RF, IGA, IGG, IGM, C3, C4, BALP, BGP, OPG, RANKL, DAS28 were all positively correlated with Sharp Score, as seen in Figure 1. 
A

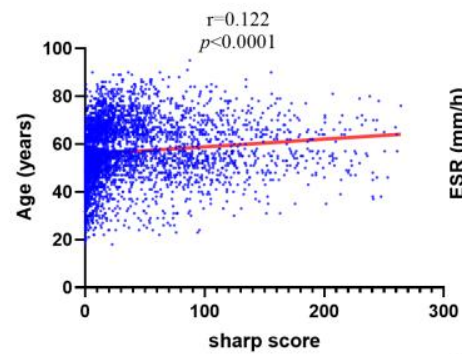

$\mathrm{D}$

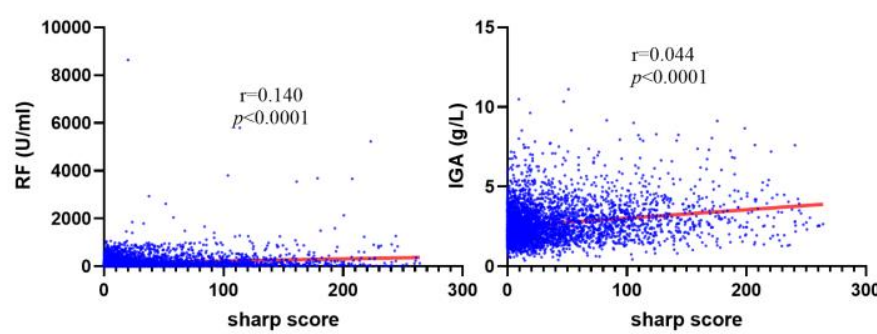

G

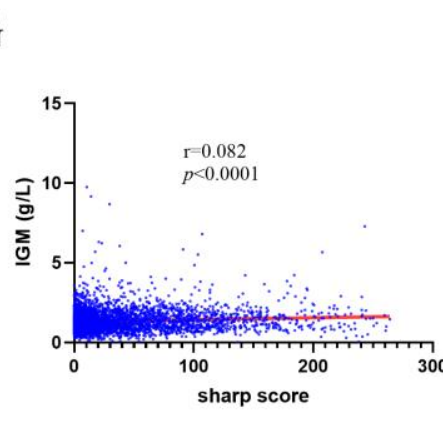

$\mathrm{J}$

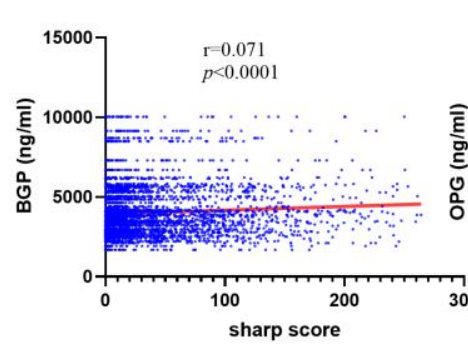

$\mathrm{H}$

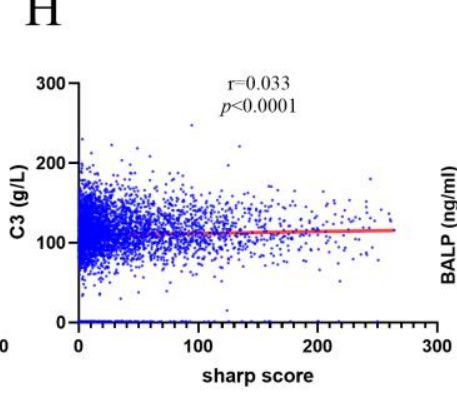

K

B

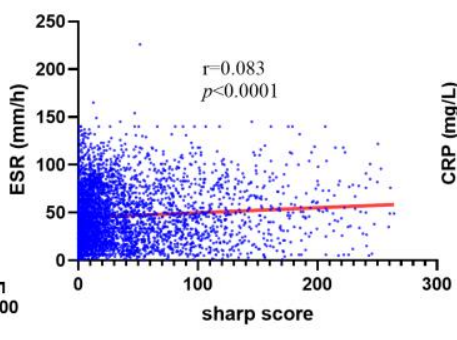

$\mathrm{E}$

$\mathrm{F}$

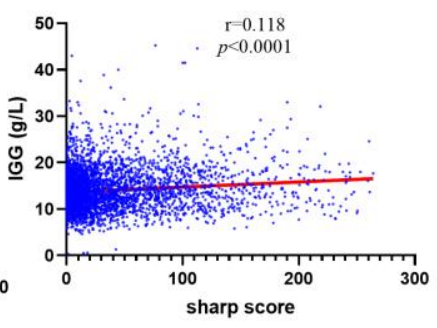

I

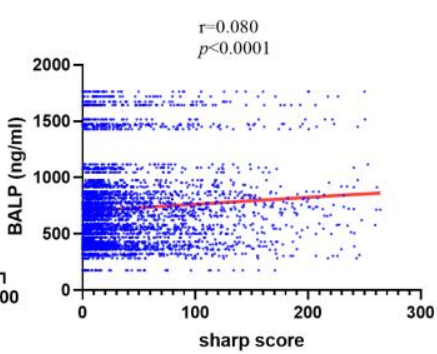

$\mathrm{L}$
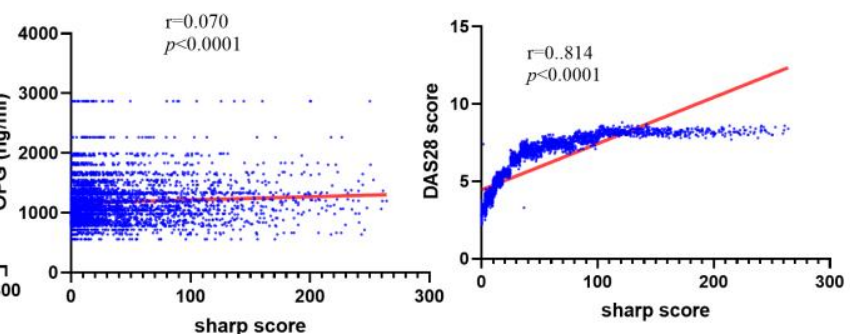

Figure 1. Spearman correlation analysis of Sharp Score and clincial indexes.

\section{Association rules analysis of Sharp Score and clincial indexes}

Association rules analysis of Sharp Score and clincial indexes can be found in Table 2. Set the minimum support to $80 \%$ and the minimum confidence to $80 \%$. Through Aprior module analysis, the correlation between Sharp Score and clincial indexes was obtained, and the degree of lift was more than 1 and $P<0.05$.

Table 2. Association rules analysis of Sharp Score and clincial indexes.

\begin{tabular}{lllll}
\hline Items $($ LHS $\Rightarrow$ RHS $)$ & Support & Confidence & Lift & P value \\
\hline
\end{tabular}




\begin{tabular}{lllll}
\hline$\{$ Sharp Score $\uparrow\} \Rightarrow\{\mathrm{ESR} \uparrow\}$ & $83.68 \%$ & $91.72 \%$ & 1.05 & $<0.01$ \\
$\{$ Sharp Score $\uparrow\} \Rightarrow\{\mathrm{CRP} \uparrow\}$ & $83.05 \%$ & $91.03 \%$ & 1.04 & $<0.01$ \\
$\{$ Sharp Score $\uparrow\} \Rightarrow\{\mathrm{RF} \uparrow\}$ & $81.25 \%$ & $91.03 \%$ & 1.04 & $<0.01$ \\
$\{$ Sharp Score $\uparrow\} \Rightarrow\{\mathrm{IGA} \uparrow\}$ & $83.05 \%$ & $87.71 \%$ & 1.05 & $<0.01$ \\
$\{$ Sharp Score $\uparrow\} \Rightarrow\{\mathrm{IGG} \uparrow\}$ & $87.67 \%$ & $87.55 \%$ & 1.05 & $<0.01$ \\
$\{$ Sharp Score $\uparrow\} \Rightarrow\{\mathrm{C} 3 \uparrow\}$ & $81.25 \%$ & $87.55 \%$ & 1.05 & $<0.01$ \\
$\{$ Sharp Score $\uparrow\} \Rightarrow\{\mathrm{BGP} \uparrow\}$ & $83.68 \%$ & $87.04 \%$ & 1.05 & $<0.01$ \\
$\{$ Sharp Score $\} \Rightarrow\{\mathrm{RANKL} \uparrow\}$ & $81.25 \%$ & $86.79 \%$ & 1.05 & $<0.01$ \\
$\{$ Sharp Score $\} \Rightarrow\{\mathrm{DAS} 28 \uparrow\}$ & $81.25 \%$ & $86.34 \%$ & 1.05 & $<0.01$ \\
\hline
\end{tabular}

\section{Logistic regression analysis of Sharp Score and clincial indexes}

Logistic regression analysis of risk factors of Sharp Score was carried out. Significant differences in Sharp Score was found between RA patients with ESR ( $p=0.000)$, $\mathrm{CRP}(p=0.023), \mathrm{RF}(p=0.000)$, indicating that ESR, CRP, RF were risk factors for Sharp Score, the higher expression of ESR, CRP, RF, the high score of sharp (Figure 2).

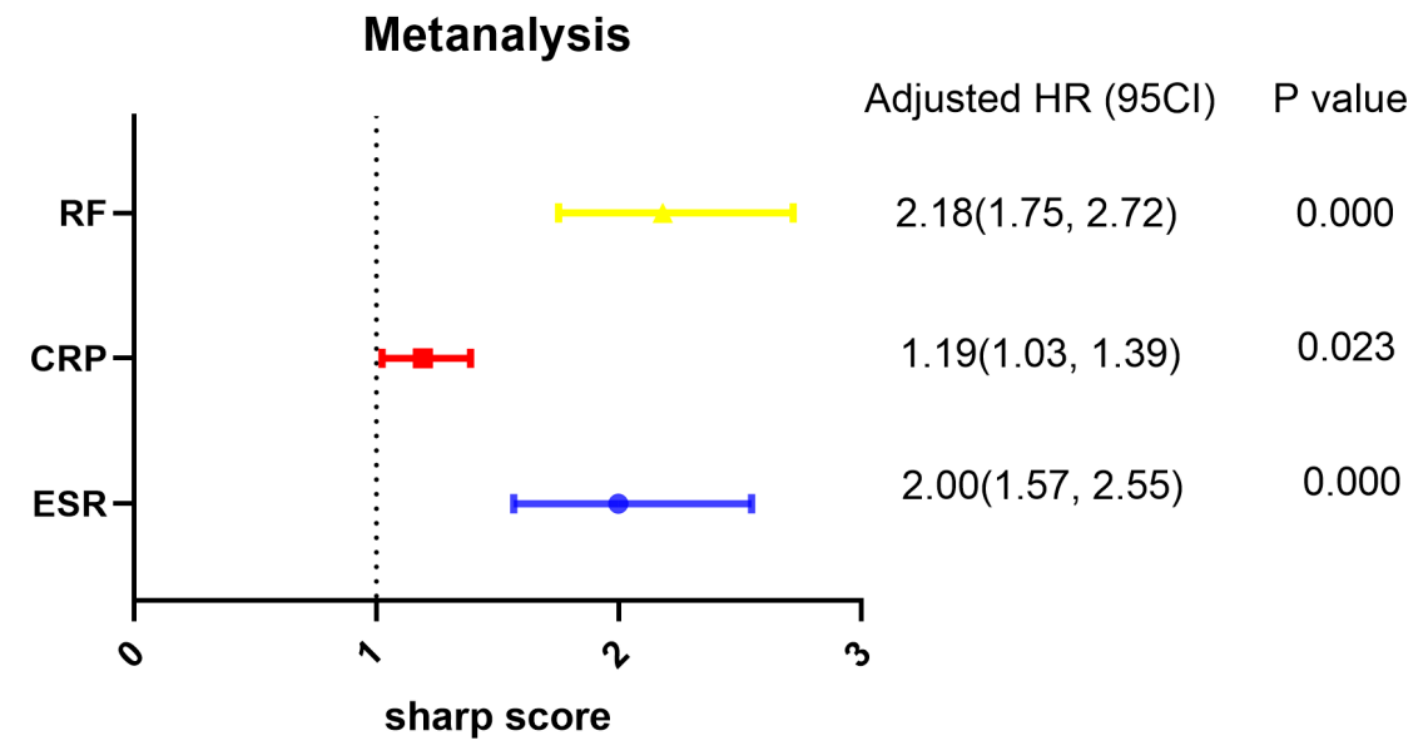

Figure 2. Logistic regression analysis of Sharp Score and clincial indexes.

\section{Comparison of Sharp Scores among different variables}

As shown in Table 3, there was a higher Sharp Score of female patients compared to male $(21.00$, IQR $(7.00,60.13)$ vs 17.00 ( IQR $(7.00,42.13))$ and a high Sharp Score of over 50 years old compared to under 50 years old ( 24.00 , IQR $(10.00,60.13)$ vs 
10.00 ( IQR $(3.00,45.00)$ ). There was a higher Sharp Score of RF - positive compared to RF-negative (23.00, IQR $(8.00,64.63)$ vs 13.00 ( IQR $(4.38,35.63)$ ). There was a higher Sharp Score of ever-smoker compared to never-smoker (20.00, IQR $(7.50,56.50)$ vs 19.50 ( IQR $(4.50,55.00))$.

Table 3. Comparison of Sharp Scores among different variables

\begin{tabular}{|c|c|c|c|c|}
\hline Quantitative Variables & Group & Sharp Score & $\mathbf{Z}$ & $P$ value \\
\hline \multirow{2}{*}{ Sex } & Female & $21.00(7.00,60.13)$ & \multirow{2}{*}{9.482} & \multirow{2}{*}{0.002} \\
\hline & Male & $17.00(7.00,42.13)$ & & \\
\hline \multirow{2}{*}{ Age } & $<50$ years & $10.00(3.00,45.00)$ & \multirow{2}{*}{154.33} & \multirow{2}{*}{0.000} \\
\hline & $\geq 50$ years & $24.00(10.00,60.13)$ & & \\
\hline \multirow{2}{*}{ Symptom duration } & $<5$ years & $5.5(0.00,1.50)$ & \multirow{3}{*}{443.97} & \multirow{3}{*}{0.000} \\
\hline & $\geq 5$ years & $25(10.50,64.00)$ & & \\
\hline \multirow{3}{*}{ DSA28 score } & $<3.2$ & $0.50(0.00,1,50)$ & & \\
\hline & $\geq 3.2<5.1$ & $6.50(3.50,10.00)$ & \multirow[t]{2}{*}{2813.43} & \multirow[t]{2}{*}{0.000} \\
\hline & $\geq 5.1$ & $47.00(25.50,89.00)$ & & \\
\hline \multirow{2}{*}{ RF } & Positivity & $23.00(8.00,64.63)$ & \multirow[t]{2}{*}{94.01} & \multirow[t]{2}{*}{0.000} \\
\hline & Negativity & $13.00(4.38,35.63)$ & & \\
\hline \multirow{2}{*}{$\mathrm{CCP}$} & Positivity & $20.00(7.00,57.38)$ & \multirow{2}{*}{3.027} & \multirow{2}{*}{0.082} \\
\hline & Negativity & $17.50(5.50,47.00)$ & & \\
\hline \multirow{2}{*}{ Ever smoker } & Yes & $20.75(4.50,55.50)$ & \multirow{2}{*}{0.996} & \multirow{2}{*}{0.318} \\
\hline & No & $19.50(7.00,56.50)$ & & \\
\hline \multirow{2}{*}{ Ever drinker } & Yes & $20.00(7.50,56.50)$ & \multirow{2}{*}{3.827} & \multirow{2}{*}{0.050} \\
\hline & No & $19.50(4.50,55.00)$ & & \\
\hline \multirow{4}{*}{ Radiographic grading of hands } & I & $1.50(0.50,2.50)$ & \multirow{4}{*}{3546.75} & \multirow{4}{*}{0.000} \\
\hline & II & $10(6.50,14.00)$ & & \\
\hline & III & $28.50(23.00,37.00)$ & & \\
\hline & IV & $87.00(61.50,122.50)$ & & \\
\hline
\end{tabular}

\section{Discussion}

This study was a large-sample retrospective study, which has characterized Sharp Score and its effective factors in RA. The role of clincial indexes, bone metabolism indexes, DAS28 and sociodemographic factors as determinants of Sharp Score was examined. Age, ESR, CRP, RF, IGA, IGG, IGM, C3, C4, BALP, BGP, OPG, RANKL, DAS28 were associated with Sharp Score. ESR, CRP, RF were also risk factors of Sharp Score.

Joint damage is very common in an early stage of RA, even within two years after 
disease onset in the vast majority $(70-93 \%)$ of patients ${ }^{17,18}$. Therefore, the probability of occurrence of erosions is adequately high in the early years of RA ${ }^{8,19}$. Therefore, joint damage may lead to the generation and maintenance of pain, which is a major cause of disability and functional decline ${ }^{20}$. In a study by Corbett et al the onset of erosions in hands during the first two years of RA was the strongest predictive feature of a poor functional outcome after 15 years $^{21}$. Early quantitative assessment of joint destruction and bone erosion are the first step to prevent or decrease its damage ${ }^{22,23}$.

Although there has not been a similar study to date describing Sharp Score and its effective factors in RA. A few studies have described Sharp Score as an important observation index and effective factor of $\mathrm{RA}^{24}$. LMAJansen followed early RA patients for one year, concluded that progression of these lesions was predicted by the number of radiographic lesions and Sharp/van der Heijde score ${ }^{12}$. Similar findings were also observed in a cross-sectional study of RA patients with secondary SS (sSS) by Lindsay E. Brown et al, which found that RA subjects with sSS had worse joint damage was associated with higher Sharp Score ${ }^{13}$. As a part of our ongoing research on the joint destruction and bone erosion, in the present study we focused on Sharp Score, which might have significant diagnostic value for RA.

Spearman correlation analysis and Association rules analysis showed that there were significant positive correlations between age, ESR, CRP, RF, IGA, IGG, IGM, C3, C4, BALP, BGP, OPG, RANKL, DAS28 and Sharp Score in our study. In addition, Logistic regression analysis presented that ESR, CRP, RF were risk factors of Sharp Score. Clincial indexes, bone metabolism indexes, DAS28 and sociodemographic factors differences in Sharp Score outcomes have not been well studied in the China and there has been no similar study on the impact of Sharp Score. There are differences in sharp score of different genders, which showed that a higher Sharp Score of female patients compared to male ${ }^{25}$. There are different explanations of these gender-based differences, which may be the both the biological progression of disease as well as self - perception and reporting of symptoms ${ }^{26}$. Higher DAS28, $\mathrm{RF}^{+}$and radiographic grading were also associated with Sharp score and this can be due 
to its association with higher levels of inflammation and comorbidities ${ }^{27,28}$. Symptom duration and smoking history could affect Sharp Score progression through differences in health literacy, self - care and medication compliance ${ }^{29,30}$.

There are several strengths in our study. First, this is the first retrospective study of Sharp Score in RA patients in China, and is uniquely placed to study the effective factors of Sharp Score in China. Furthermore, to the best of our knowledge, this is the only study to date that has used three methods to identify a significant correlation between Sharp Score and different variables. One of the limitations of our study was the lack of multi-center and inclusion of diverse ethnic/racial groups. Furthermore, our research size is small, and this may limit finding significant differences between Sharp Score and different variables. Additionally, we also need to study the diagnostic accuracy and importance of magnetic resonance imaging and take it into the next research.

In conclusion, the study has characterized the association between Sharp Score and clincial indexes, bone metabolism indexes, DAS28 and sociodemographic factors, and demonstrated that a significant correlation between Sharp Score and these variables. The demonstrated differences in joint damage and bone erosion in the early course of RA may have an impact on subsequent long - term outcomes. Disparities in Sharp Score should be taken into consideration in formulating treatment strategies in the early course of the disease, when intervention is most likely to benefit the patient. This is essential in improving disease control and functional outcomes.

\section{Conclusions}

In conclusion, the Sharp Score in patients with RA was significantly increased and closely related to disease activity. In addition to that, ESR, CRP and RF were risk factors of Sharp Score. So, Sharp Score should be taken into consideration in formulating treatment strategies in RA.

\section{Abbreviations}

RA: rheumatoid arthritis; DAS28: Disease Activity Score; CRP: C-reactive protein; 
RF: rheumatoid factor; ESR: erythrocyte sedimentation rate; CCP: anti-cyclic citrullinated peptide antibody; IGA: immunoglobulins A; IGG: immunoglobulin G; IGM: immunoglobulin M; C3: complement 3; C4: complement 4; BALP: bone alkaline phosphatase; OC: osteocalcin; OPG: Osteoprotegerin; RANKL: receptor activator for nuclear factor- $\mathrm{\kappa} \mathrm{B}$ ligand; ARM: association rule mining.

\section{Acknowledgements}

Not applicable.

\section{Authors' contributions}

JTW, JL, HJ, LW, LX, and YS contributed to the study design. JTW contributed to data analysis, wrote the first draft, and revised the manuscript. YQS, XW, and JW contributed to the questionnaire survey on patients, specimens, and data collection. JL supervised the project and helped revise the manuscript. All authors reviewed and accepted the content of the final manuscript.

\section{Fundings}

This work was supported by grants from Ministry of Science and Technology National Key Research and Development Program Chinese Medicine Modernization Research Key Project (2018YFC1705204); National Nature Fund Program (81973655); The Key Research and Development Program Foreign Science and Technology Cooperation Project of Anhui (201904b11020011); Anhui Provincial Quality Engineering Teaching and Research Project (2018jyxm1068); Anhui Famous Traditional Chinese Medicine Liu Jian Studio Construction Project (Traditional Chinese Medicine Development Secret [2018] No. 11); National Key innovative talents training Project of Traditional Chinese Medicine (National Education letter of Traditional Chinese Medicine [2019] No.128); key Research and Development Plan Project of Anhui Province (201904a07020004); Anhui Provincial Laboratory of Applied basis and Development of Internal Medicine of Modern Traditional Chinese Medicine (2016080503B041); 12th batch of "115" Innovation team of Anhui Province 
(Anhui Talent Office [2019] No. 1).

\section{Availability of data and materials}

The raw data supporting the conclusions of this article will be made available by the authors, without undue reservation to any qualified researcher.

\section{Ethics approval and consent to participate}

The study was approved by the Ethics Committee of the First Affiliated Hospital of Anhui University of Traditional Chinese Medicine and carried out under the Helsinki Declaration. Before participating in the study, the patients filled in a written informed consent forms. A written informed consent was obtained from all the study participants.

\section{Consent for publication}

Not applicable.

\section{Competing interests}

The authors declare that they have no competing interests.

\section{Author details}

${ }^{1}$ Anhui University of Traditional Chinese Medicine, Hefei 230031, Anhui Province, China. ${ }^{2}$ Department of Rheumatology and Immunology, First Affiliated Hospital of Anhui University of Traditional Chinese Medicine, Hefei 230038, Anhui Province,

China. ${ }^{3}$ Institute of Rheumatology, Anhui Academy of Traditional Chinese Medicine, Hefei 230012, Anhui Province, China.

\section{References}

1 Smolen, J. J. J. o. a. Insights into the treatment of rheumatoid arthritis: A paradigm in medicine. 110, 102425, doi:10.1016/j.jaut.2020.102425 (2020). 
Small, A. \& Wechalekar, M. J. E. r. o. m. d. Synovial biopsies in inflammatory arthritis: precision medicine in rheumatoid arthritis. 20, 315-325, doi:10.1080/14737159.2020.1707671 (2020).

3 Boissier, M., Semerano, L., Challal, S., Saidenberg-Kermanac'h, N. \& Falgarone, G. J. J. o. a. Rheumatoid arthritis: from autoimmunity to synovitis and joint destruction. 39, 222-228, doi:10.1016/j.jaut.2012.05.021 (2012).

4 Mclnnes, lain, B., Schett \& Medicine, G. J. N. E. J. o. The Pathogenesis of Rheumatoid Arthritis. (2011).

5 (!!! INVALID CITATION !!! 5).

6 Combe, B. et al. 2016 update of the EULAR recommendations for the management of early arthritis. 76, 948-959, doi:10.1136/annrheumdis-2016-210602 (2017).

7 Schett, G. \& Gravallese, E. J. N. r. R. Bone erosion in rheumatoid arthritis: mechanisms, diagnosis and treatment. 8, 656-664, doi:10.1038/nrrheum.2012.153 (2012).

8 Fuchs, H., Kaye, J., Callahan, L., Nance, E. \& Pincus, T. J. T. J. o. r. Evidence of significant radiographic damage in rheumatoid arthritis within the first 2 years of disease. 16,585-591 (1989).

9 Zijlstra, T., Bernelot Moens, H. \& Bukhari, M. J. A. o. t. r. d. The rheumatoid arthritis articular damage score: first steps in developing a clinical index of long term damage in RA. 61, 20-23, doi:10.1136/ard.61.1.20 (2002).

Kowalski, E. \& Chung, K. J. H. Impairment and disability: Renoir's adaptive coping strategies against rheumatoid arthritis. 7, 357-363, doi:10.1007/s11552-012-9467-4 (2012).

Quintana-Duque, M. et al. Predictors of remission, erosive disease and radiographic progression in a Colombian cohort of early onset rheumatoid arthritis: a 3-year follow-up study. 35, 1463-1473, doi:10.1007/s10067-016-3246-5 (2016). Jansen, L., van der Horst-Bruinsma, I., van Schaardenburg, D., Bezemer, P. \& Dijkmans, B. J. A. o. t. r. d. Predictors of radiographic joint damage in patients with early rheumatoid arthritis. 60, 924-927, doi:10.1136/ard.60.10.924 (2001).

Brown, L. et al. Clinical characteristics of RA patients with secondary SS and association with joint damage. 54, 816-820, doi:10.1093/rheumatology/keu400 (2015). Mochizuki, T. et al. Correlation between hand bone mineral density and joint destruction in established rheumatoid arthritis. 14, 461-465, doi:10.1016/j.jor.2017.07.010 (2017). D, A. et al. 2010 Rheumatoid arthritis classification criteria: an American College of Rheumatology/European League Against Rheumatism collaborative initiative. 62, 2569-2581, doi:10.1002/art.27584 (2010).

16 Xie, J. et al. Association learning of Chinese herbal medicines and disease treatment efficacy. 1-20 (2018).

Möttönen, T., Hannonen, P., Toivanen, J., Rekonen, A. \& Oka, M. J. A. o. t. r. d. Value of joint scintigraphy in the prediction of erosiveness in early rheumatoid arthritis. 47, 183-189, doi:10.1136/ard.47.3.183 (1988).

Wick, M., Lindblad, S., Klareskog, L. \& Van Vollenhoven, R. J. A. o. t. r. d. Relationship between inflammation and joint destruction in early rheumatoid arthritis: a mathematical description. 63, 848-852, doi:10.1136/ard.2003.015172 (2004). 

damage over 10 years in a cohort with early rheumatoid arthritis. 62, 611-616, doi:10.1136/ard.62.7.611 (2003).

Závada, J. et al. The relationship between synovitis quantified by an ultrasound 7-joint inflammation score and physical disability in rheumatoid arthritis - a cohort study. 19, 5 , doi:10.1186/s13075-016-1208-6 (2017).

Young, A., Bielawska, C., Corbett, M. \& Roitt, I. J. C. r. A prospective study of early onset rheumatoid arthritis over fifteen years: prognostic features and outcome. 12-19, doi:10.1007/bf02203380 (1987).

Jawaid, W., Crosbie, D., Shotton, J., Reid, D. \& Stewart, A. J. A. o. t. r. d. Use of digital x ray radiogrammetry in the assessment of joint damage in rheumatoid arthritis. 65, 459-464, doi:10.1136/ard.2005.039792 (2006).

Klarlund, M. et al. Magnetic resonance imaging, radiography, and scintigraphy of the finger joints: one year follow up of patients with early arthritis. The TIRA Group. 59, 521-528, doi:10.1136/ard.59.7.521 (2000).

Forslind, K. et al. Hand bone loss measured by digital X-ray radiogrammetry is a predictor of joint damage in early rheumatoid arthritis. 38, 431-438, doi:10.3109/03009740902939376 (2009). van Vollenhoven, R. J. B. m. Sex differences in rheumatoid arthritis: more than meets the eye. 7, 12, doi:10.1186/1741-7015-7-12 (2009). Sokka, T. et al. Women, men, and rheumatoid arthritis: analyses of disease activity, disease characteristics, and treatments in the QUEST-RA study. 11, R7, doi:10.1186/ar2591 (2009). Yang, X., Chang, Y. \& Wei, W. J. M. o. i. Endothelial Dysfunction and Inflammation: Immunity in Rheumatoid Arthritis. 2016, 6813016, doi:10.1155/2016/6813016 (2016). DeMizio, D., Geraldino-Pardilla, L. J. R. \& therapy. Autoimmunity and Inflammation Link to Cardiovascular Disease Risk in Rheumatoid Arthritis. 7, 19-33, doi:10.1007/s40744-019-00189-0 (2020). Khoo, H., Lim, Y. \& Vrijhoef, H. J. A. P. f. m. Primary healthcare system and practice characteristics in Singapore. 13, 8, doi:10.1186/s12930-014-0008-x (2014). Behaviors in Rheumatoid Arthritis Patients. 15, E127, doi:10.5888/pcd15.180218 (2018). 
A

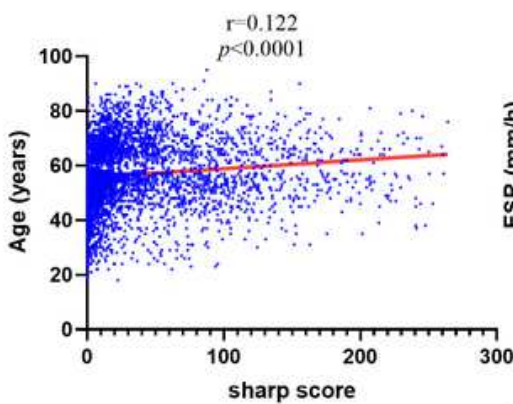

D

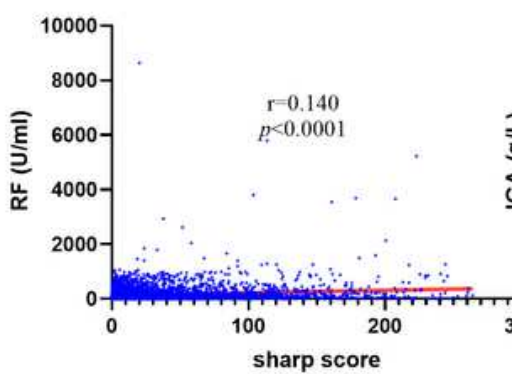

G

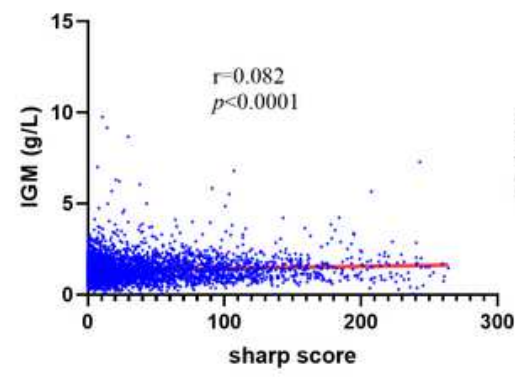

$\mathrm{J}$

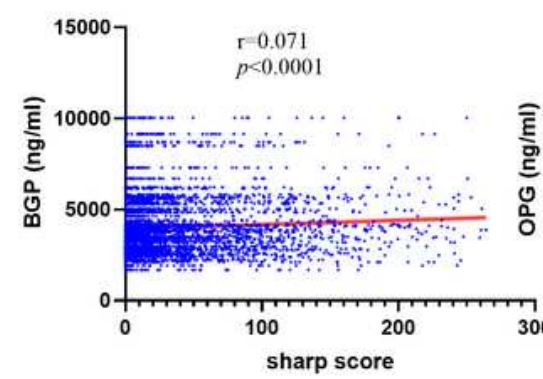

B

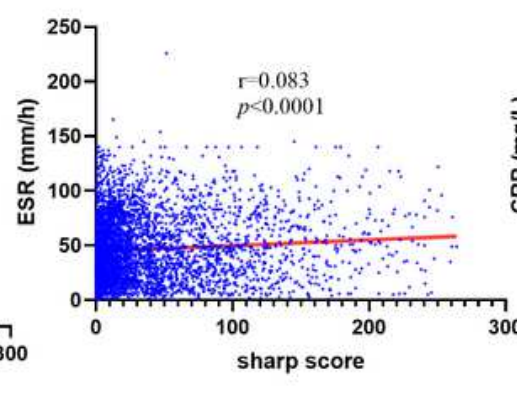

$\mathrm{E}$

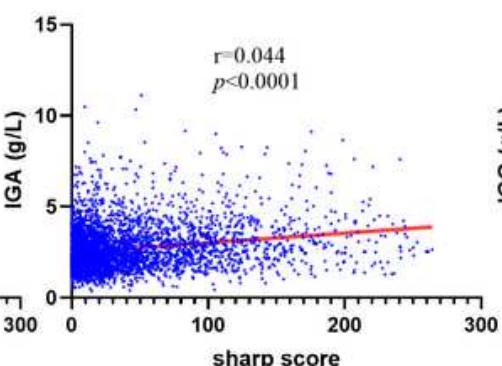

$\mathrm{H}$

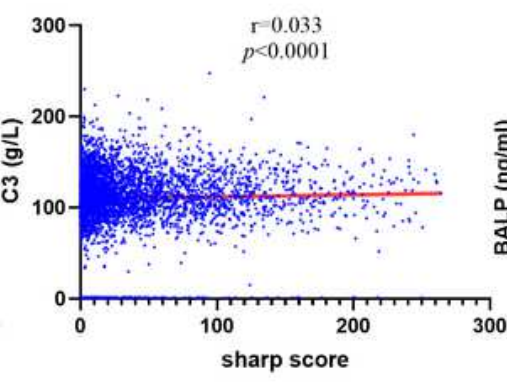

$\mathrm{K}$
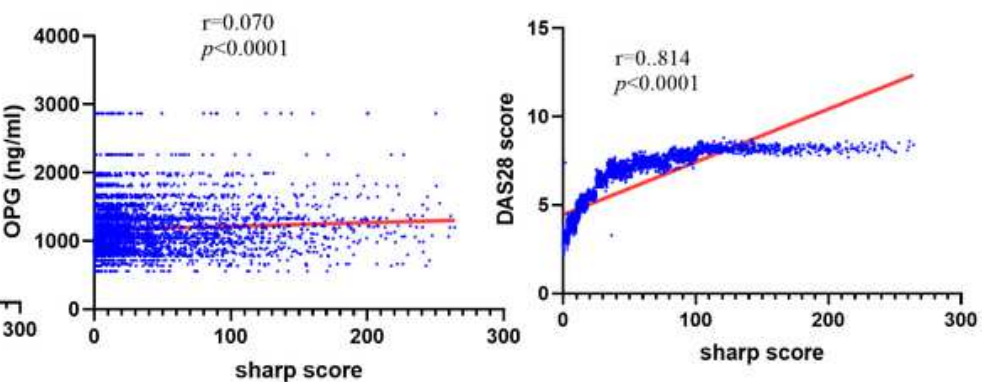

Figure 1

Spearman correlation analysis of Sharp Score and clincial indexes. 


\section{Metanalysis}

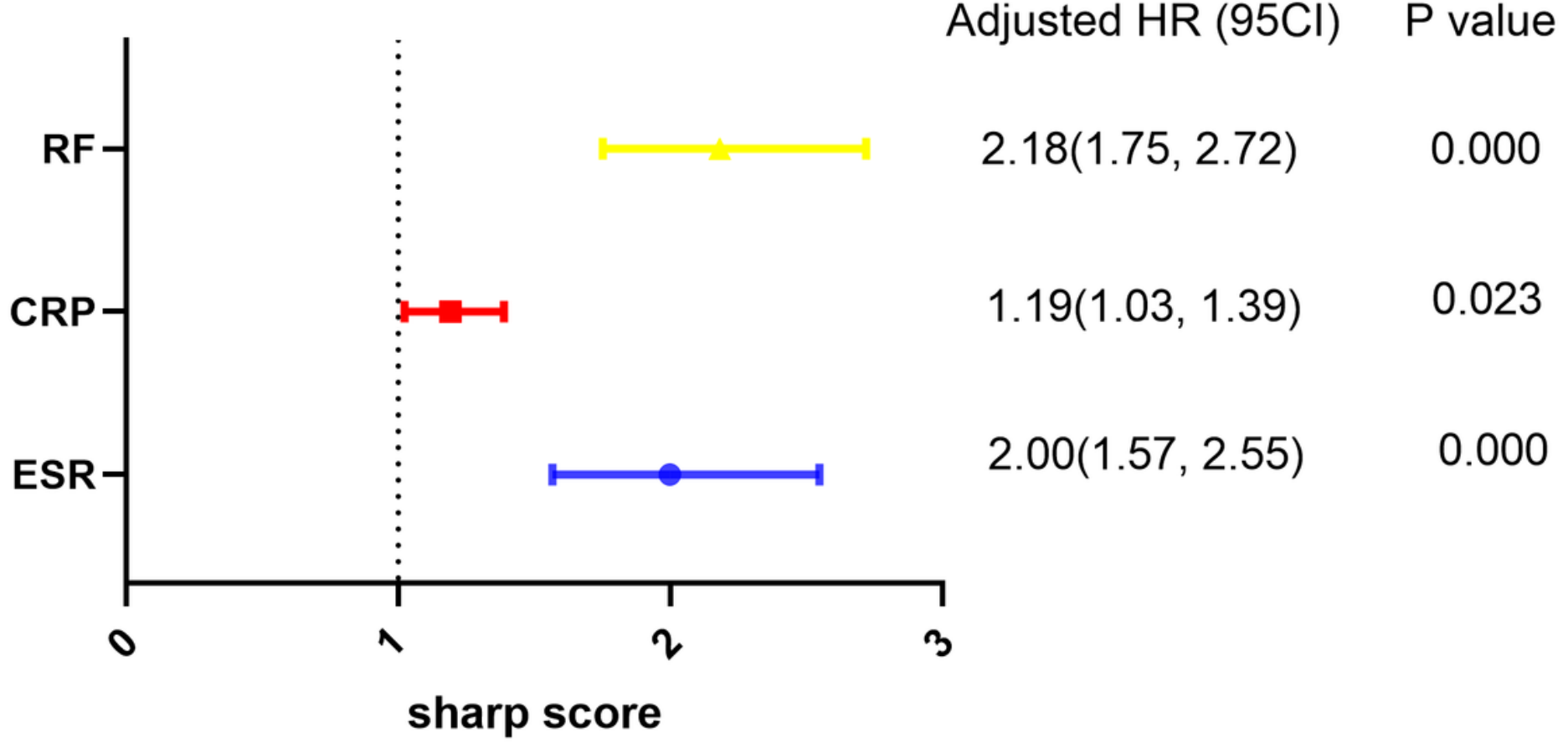

Figure 2

Logistic regression analysis of Sharp Score and clincial indexes. 